\title{
La femme maghrébine et française d'origine maghrébine confrontée aux préjugés de la société française
}

\section{The Maghrebian and French woman of Maghreb origin confronted with the prejudices of French society}

\author{
NaIma Benaicha ZIANI \\ naima.benaicha@ua.es \\ AzUCENA ORTIZ García \\ azucena.ortz@gmail.com \\ Universidad de Alicante
}

\begin{abstract}
France, the founding country of the Human Rights, also known as 'Host Land', has received significant migratory flows throughout its history, originating from its former colonies in the Maghreb. In its various generations, Maghreb women are a key element in the overall development of French society. However, the Hexagon has always been reluctant to accept this multiculturalism. Hence, these women are considered as foreigners in their double sense: either they come from outside, or they are strange. This feeling is based on a series of prejudices and stereotypes that gravitate to the French collective subconscious. Thus, this article aims to show the continuing validity of the problematic on French identity.
\end{abstract}

Key-words

Identity, prejudices, stereotypes, foreign, strange.

\begin{abstract}
Resumen
Francia, el país fundador de los Derechos del Hombre, también conocido como 'Tierra de acogida', ha recibido a lo largo de su historia importantes flujos migratorios, provenientes de sus antiguas colonias del Magreb, siendo la mujer magrebí, en sus diversas generaciones, un elemento clave para desarrollo integral de la sociedad francesa, Sin embargo, el Hexágono siempre ha sido reticente para aceptar esta multiculturalidad. De ahí, que estas mujeres estén consideradas como extranjeras en su doble sentido, es decir, bien que vienen de fuera, bien que son extrañas, en base a una serie de prejuicios y estereotipos, que gravitan en el subconsciente colectivo francés. Así, este artículo pretende mostrar la todavía vigencia de la problemática sobre la identidad francesa.
\end{abstract}

\section{Palabras clave}

Identidad, prejuicios, estereotipos, extranjera, extraña. 


\section{Introducción}

Avant d'entamer notre étude, nous tenons à mettre en exergue que la culture française n'est pas que Molière, car dès le XIX ${ }^{\mathrm{e}}$ siècle, Napoléon déclencha son aventure colonialiste en Egypte et les échanges culturels entre Orient et la France se multiplia de manière très productive pour les deux civilisations. D'un côté, l'Égypte avec le Liban, la Syrie et d'autres pays arabes, et plus tard ceux du Maghreb, se sont bénéficié de la modernité de la science et de la technologie occidentale, en plus des apports de la presse, pour mener à terme leur renaissance culturelle dans les Lettres et la pensée arabe, moyennant le courant rénovateur dénommé, Nahda ${ }^{1}$. De l'autre côté, la France éprouva ces intellectuels, futures élites de ces pays, avides d'acquérir de nouvelles connaissances et en même temps partager avec les connaissances françaises leurs savoirs millénaires. Par conséquent, l'esprit français allait se nourrir, même à son insu, de ces richesses culturelles des peuples et des civilisations conquises, tout au long de l'Histoire, notamment, celles du Maghreb. C'est pourquoi, la question sur l'identité a été et continue à être un enjeu à aboutir par la nation française, car, le pays des droits de l'homme a ancré dans le plus profond de soi, le mot contradiction. Or, dans cet oxymore, compris entre la tradition et la Révolution, qui est la France, celle-ci a largement négligé la diversité comme un élément fondamental de son idiosyncrasie. Jean d'Ormesson le défini si bien: "Être français, c>est aimer la tradition et c>est aimer la Révolution. Être français est d〉abord une contradiction". ${ }^{2}$ Cela étant dit, il ne nous reste plus qu'à rappeler l'importance de la contribution de la culture maghrébine dans le concept d'identité française. À notre avis, elle a été systématiquement ignorée, méprisée et pas suffisamment prise en compte par l'ensemble de la société française, bien que sa culture soit faite, spécialement, du rassemblement de ces contrastes. En effet, Simona Tersigni (2001: 55-65) souligne que la réticence envers la culture maghrébine est due à la confrontation du mythe de l'homogénéité française, à l'égard du discours normatif nationale sur l'intégration des Maghrébins. D'une part, à cause de l'idée erronée du partage d'un code d'honneur propre à tous les migrants originaires du Maghreb, et de l'autre, en vertu de l'association implicite entre musulmanes et Arabes, en tant que sujets inadmissibles par rapport au modèle républicain. Cette contribution est, donc, un hommage à la culture maghrébine, comme un élément incontournable de l'identité française, propre à ses contradictions et à sa diversité intrinsèque. En somme, emportées par ce caractère contradictoire de l'Hexagone puisque, dans son hétérogénéité, il se trouve qu'il y a toujours, une invitation continuelle aux débats constructifs, favorisant ainsi les échanges avec

1 Mot que l'on peut traduire par 'essor' ou 'force'. Pour plus de renseignements, consulter: < https://www. monde-diplomatique.fr/mav/106/DUPONT/17685> [22/05/2021].

2 Pour plus de renseignements sur l'idée de l'académicien, écrivain et philosophe français, Jean d'Ormesson, consulter: <https://www.lepoint.fr/societe/etre-francais-selon-jean-d-ormesson-13-01-2011-129480_23.php> [14/05/2021]. 
“ces autres”, en l'occurrence, les Maghrébins ou les Français d'origine maghrébine, afin de réussir une reconnaissance et un respect mutuels, dans leur cohabitation harmonieuse, au sein de la complexité du concept identitaire français.

En outre, les courants migratoires ne sont plus une tendance, mais une réalité palpable dans ce contexte de plus en plus globalisé. Incontestablement, les dernières statistiques sur la migration dans l'Union Européenne démontrent que 45\% des migrants sont des femmes. Par ailleurs, l'expérience de l'immigration et de l'intégration dans le pays de résidence peut être vécue de manière différente aussi bien par les hommes que par les femmes. Les discriminations subies peuvent être également différentes en raison du genre. Mais, de qui nous parlons lorsque nous évoquons les femmes d'origine maghrébine en France? Dans l'expression femmes immigrées, il y a deux typologies: L'une renvoie aux femmes non européennes, provenant des pays du Maghreb, celles de la première génération d'immigrés et l'autre fait référence aux femmes issues des familles immigrées, nées dans le pays de résidence de leurs parents, appartenant à la deuxième, troisième et même quatrième génération ${ }^{3}$.

Par ailleurs, l'objectif de cet article est de mettre l'accent sur la problématique de la situation de la femme maghrébine ou d'origine maghrébine qui vit en France, afin de réfléchir à la question, par excellence encore non résolue, celle de l'identité. Pour déchiffrer cette énigme, nous émettons les interrogations suivantes: Qu'est-ce que c'est être français? Quels sont les éléments qui définissent l'identité française? Peut-on parler de catégories différentes dans le statut de citoyenne française? Quels sont les obstacles que ces femmes doivent franchir, dans la société et dans leur entourage familial, afin de s'imposer en tant que française? En d'autres termes, comment la femme d'origine maghrébine est considérée, d'après le contraste fondamentale, entre l'image qu'elle a de soi-même et l'image qu'elle renvoie aux autres, non seulement aux citoyens français "de souche" à ses congénères, concernant ses rôles et sa place dans la société, dont elle fait partie, à part entière et de manière engagée, en tant que sujet individuel, singulier et actif, malgré les clichés et les stéréotypes, auxquels elle est réduite en raison de son origine (Le Bras, 2017: 68-71).

3 Ce chiffre est cité dans le Rapport sur la situation des femmes issue de groupes minoritaires dans l'Union européenne. Commission des droits de la femme et de l'égalité des chances, Parlement européen, A-5 0102/2004: $<$ https://www.europarl.europa.eu/sides/getDoc.do?pubRef=-//EP//TEXT+REPORT+A5-2004-0102+ $0+\mathrm{DOC}+\mathrm{XML}+\mathrm{V} 0 / / \mathrm{FR}>[12 / 06 / 2021]$.

4 Nous avons tenu à mettre cette expression entre guillemets pour attirer l'attention du lecteur et l'inviter à réfléchir sur l'interrogation suivante: qui, de nos jours, est Français de souche? 


\section{La question de genre et les fondements de la culture maghrébine chez la femme maghrébine et française d'origine maghrébine: Les enjeux à affronter dans la société française}

Comme nous l'avons déjà souligné, le thème qui nous occupe dans cet article est d'une ampleur suffisamment importante sur le plan des politiques d'intégration et identitaire du collectif féminin maghrébin en France ainsi que des femmes françaises d'origine maghrébine. Avant de nous aventurer dans l'analyse de ce sujet, nous insistons à reconnaître qu'aborder de tels éléments supposerai de pouvoir maîtriser un ensemble de connaissances historiques, sociales, anthropologiques et mêmes linguistiques réservées aux spécialistes dans la matière. Nous allons, donc, commencer par expliquer les sujets principaux abordés le long de cet article, portant sur divers aspects relatifs à la condition de la femme maghrébine et de la femme française d'origine maghrébine en France.

\subsection{La problématique identitaire et de genre chez la femme d'origine maghrébine}

La construction socioculturelle de l'identité, selon la conception sociale et individuelle chez la femme d'origine maghrébine, que ce soit les migrantes de la première génération, arrivant en France dans les années 70, ou les descendantes de celles-ci, connues sous le nom de "beurettes" nées en France, et par conséquent, ayant la nationalité française acquise par le "droit du sol"5, oscille entre deux mondes tout à fait opposés: l'oriental liée à la sauvegarde des traditions et de la mémoire et l'occidental, mis en rapport avec la multiplicité de nouvelles références culturelles à leur portée, dans la société d'accueil. C'est ainsi que, dans ce processus d'adaptation en France, il n'y a qu'un seul paradigme à suivre, de sorte que ces femmes doivent négocier de manière simultanée la socialisation interculturelle avec la construction de leur identité personnelle, tout en affrontant la problématique de l'acculturation. Par ailleurs, la représentation fortement stéréotypée de la femme d'origine maghrébine, de la part des Européens, focalisée sous une forte vision ethno centrée, concentrée notamment dans des aspects issus du folklore populaire, voire la musique, la gastronomie, les fêtes, la langue, ceux de l'apparence physique et du code vestimentaire associé à la pratique religieuse, notamment la portée ou non du voile islamique ou hijab, en plus des rôles traditionnels associés en raison de son genre, voire la maternité et la transmission aux filles de l'héritage des traditions à respecter, ajoute-elle des contraintes à ces complexes dynamiques identitaires au sein du contexte socioculturel français, où elle en fait partie (Qribi, 2017: 2).

En effet, dans cette socialisation, comprise comme le modelage des individus au

5 Historiquement, l'attribution de la nationalité française repose sur deux grands principes: le droit du sang et le droit du sol. Pour plus de renseignements, consultez: <https://www.vie-publique.fr/nationalite-francaise> $[12 / 06 / 2021]$. 
moyen d'une inculcation des manières d'être, de penser et de sentir spécifiques à une société ou un groupe social, il peut y avoir deux situations hétérogènes, dont la première est l'acculturation qui suppose la rencontre de deux ou plusieurs groupes porteurs de traditions culturelles différentes. Cet élément est basé sur trois modèles: le premier est celui de la contre-acculturation, caractérisé par la prise d'une position défensive afin de protéger l'identité originelle, éprouvé comme fragile à cause de l'exil. Le deuxième, le syncrétique, suppose la réalisation des choix culturels de manières diverses et sans une logique, d'où le resurgissement de contradictions, parfois insurmontables. Et le troisième, le synthétique qui comporte une combinaison cohérente entre la tradition et la modernité. On peut ainsi dire que l'acculturation est propre aux femmes maghrébines de la première génération d'immigrés, provenant des pays du Maghreb, qui, à leur arrivée en France, font la découverte des modes de vie occidentaux basés sur l'individualisme opposé au caractère hégémonique dans les sociétés du monde maghrébin (Qribi, 2017: 3). La deuxième situation est l'interculturation. Celle-ci est relative aux "beurettes", femmes maghrébines appartenant à la deuxième génération. Cette situation conçoit une dynamique à la fois, d'acculturation, c'est-à-dire, le choc avec la culture d'origine et d'appropriation de la nouvelle culture dans un contexte divers où parfois les contradictions sont difficiles à gérer surtout chez les jeunes filles qui réussissent à développer leur créativité, afin de prendre leur destin en main. Or, dans la configuration de l'identité, visant la réalisation personnelle, il y a une troisième formule, outre ces deux modèles mentionnés plus haut. Il s'agit de l'identisation qui consiste à trouver l'équilibre dans l'adéquate gestion des contradictions culturelles, d'après un triple axe, voire le contrôle des contraintes objectives, l'adoucissement des conflits subjectifs et la sauvegarde de son individualisme. Cette typologie est inscrite dans la dynamique globale de la personnalisation qui engage la personnalité du sujet féminin dans la dimension collective de la société à laquelle elle appartient (Lacoste Dujardin, 2010).

Quant à la question de genre, celle-ci est conçue dans toutes les sociétés, y compris la maghrébine, en fonction du sexe biologique. Ainsi, la femme reste subordonnée à une place inférieure dans ses rôles et son statut par rapport à l'homme, car elle est réduite à une activité non rétribuée dans le foyer, notamment, la réalisation en exclusivité des tâches ménagères, des soins des enfants et d'autres personnes dépendantes de l'entourage familial, voire les parents âgés, les beaux-parents ou personnes handicapées. Cependant, si elle contribue aux revenues de la famille, ceux-ci proviennent des tâches complémentaires au travail rémunéré de l'homme, représentant le socle principal du soutien économique familial. Par conséquent, l'homme a une position de pouvoir, par rapport à la femme, non seulement économiquement, mais aussi du point de vue des rôles dans la société, où il jouit d'une liberté d'action, aidant le progrès, nié à la femme en raison du rôle traditionnel de soigneuse qu'elle doit absolument respecter, afin de ne pas déstabiliser le système capitaliste. Il est à supposer que c'est la mère qui est la responsable de transmettre à ses filles leur destin incontournable, en raison de leur 
condition de femme, ancrée, en particulier, sur la soumission totale, de grands efforts de ce qui sera attendu d'elle, où ses vertus de bonne conduite, de bonne ménagère, puis de mère de garçon sont son seul recours et sa seule raison d'être. En conséquence, de cette dynamique de genre se dégagent les clichés occidentaux sur l'image de la femme Maghrébine. Or, cette conception n'est pas inamovible puisque, dans cette tâche de configuration de l'identité de genre, il y a plusieurs facteurs qui interagissent, voire, l'appropriation des rôles d'homme de la part de la femme, les facteurs sociaux, culturels et aussi, psychologiques. À cet effet, Lacoste souligne que la femme maghrébine ne se contente, donc, pas d'avoir une attitude passive ou réactive face aux diverses influencent, dont elle en est l'objet, mais elle leur donne sens à travers ses expériences personnelles en interaction avec son contexte de socialisation (Qribi, 2017: 3-4).

La construction de genre et de l'identité de la femme d'origine maghrébine, aussi bien la première que la deuxième génération, se développe dans les échanges socioculturels pluriels, malgré les héritages familiaux, tout en affrontant un processus complexe d'adaptation, en fonction de sa personnalité et ses projets. Ce travail de réajustement est étroitement lié à une conception idéale de soi, qui prend sens dans cette dynamique globale de personnalisation, par le biais de sa participation active et engagée dans la société (Mozzo Counil, 1994: 20-22).

\subsection{Les traditions: Le code social, l'Islam et la famille}

La tradition, la doctrine et la pratique religieuse ou morale se transmettent à travers la parole ou l'exemple dans l'étendue de l'idiosyncrasie arabo-musulmane. Concernant le type de foi à embrasser parmi cette communauté, il n'est pas facile d'identifier ce qui vient de la religion islamique et ce qui vient de la culture berbéro-arabe, etc. Or, il est possible de repérer de micro-cultures, d'après chaque région, avant la conquête de l'Islam. Mais, c'est à partir de l'islamisation ${ }^{6}$ du Maghreb que le Coran et les hadiths ${ }^{7}$ conforment leurs consignes immuables à suive. De cette manière, la tradition en Islam constitue pour le Maghreb un modèle culturel idéal à suivre pour les croyants, puisque s'y soumettre assure d'être dans le salut de Dieu, tandis que l'éloignement suppose la perdition et l'erreur. Cette tradition est devenue encore plus forte comme signe d'identité, en dépit des contraintes historiques, voire, la colonisation et l'introduction du capitalisme, propres du monde occidental. Elle repose sur un corpus de règles, des codes et de lois issues du Coran. C'est pourquoi ce Livre Saint régit tous les aspects de la vie privée comme la vie sociale et représente le guide essentiel qui oriente la vie quotidienne, car, il est à la foi, la Bible ${ }^{8}$, comme

6 En religion, c'est le fait d'islamiser, de convertir à l'Islam et ce qui en résulte. Fait d'appliquer la loi islamique. Pour plus de renseignements, consulter: $<$ www.universalis.net $>[12 / 06 / 2021]$.

7 Ensemble de traditions relatives aux comportements (actes et paroles) du Prophète Mohammad (Mahomet).

8 Il ne s'agit pas, bien évidemment, du livre Saint des chrétiens, mais du Coran comme livre sacré des musulmans. 
fondement religieux et le Code Civil comme ordonnateur juridique et social. À cet égard, la structure familiale et les rôles entre ses membres, y compris la discrimination homme/ femme y sont bien définis dans une hiérarchie spécifique. De sorte que la structure traditionnelle de base est conçue d'après la famille élargie. Pour la femme, le mariage marque la rupture avec sa famille pour "appartenir" à celle de son mari; elle va se trouver alors placée sous l'autorité de sa belle-mère. Dans ce cadre traditionnelle familiale, la femme a le statut de mère et de gardienne des traditions, en étant aussi responsable de leur transmission à leurs filles qui apprennent, depuis leur plus tendre enfance, à garder une position soumise par rapport à l'homme, selon les préceptes du Coran, puisque, l'honneur de celui-ci dépend principalement de la bonne conduite morale des femmes de la famille, dont la chasteté, la fidélité et la continence en sont des piliers fondamentaux. Alors, le rôle de la femme à la maison est imposé par l'homme et la modification de ce statut ménager risque de générer un déséquilibre des bases patriarcales ou familiales sur lesquelles se fondent ces sociétés. De même, l'Islam reconnaît à la femme des droits et des devoirs, mais dans une sphère limitée, notamment, elle peut garder son nom paternel après le mariage; le douaire ${ }^{9}$ lui appartient; la répudiation est limitée par l'introduction d'une période d'attente de trois mois; elle peut hériter ou posséder des biens librement; elle est soumise à l'acceptation de la polygamie, etc. (Wijdan, 2002: 95). De nos jours, cette situation traditionnelle se produit en milieu rural au Maghreb et dans quelques familles en immigration, surtout, celles appartenant à la première vague, à partir de la Loi du regroupement familiale de 1974 promulguée par le Président de la République Française à l'époque, Jacques Chirac. Nonobstant, dans la dynamique de changements sociales liées aux processus d'industrialisation, la famille maghrébine traditionnelle a évolué vers d'autres modèles, à savoir, la famille nucléaire ${ }^{10}$, et la famille monoparentale ${ }^{11}$, issue de mères divorcées qui prennent en charge, seules, le maintien de la structure familiale (Guerraoui \& Sturm, 2012: 291).

\subsection{Les stéréotypes chez l'image de la femme d'origine maghrébine}

En attendant, la femme d'origine maghrébine est traditionnellement associée à divers clichés, issus de ses caractéristiques physiques typiquement méditerranéennes; son apparence liée à un code vestimentaire déterminé; son nom et prénom révélant ses racines géographiques et ses rôles précis, déterminés par les traditions, tout en l'englobant dans une catégorie restreinte, en dépit de l'individualité de chaque sujet sans prendre en compte la diversité de ses particularités, surtout, si l'on considère sa provenance des mariages mixtes, en d'autres termes, ceux qui se composent de l'union entre des conjoints: l'un d'origine

9 Sorte de dot versée par le fiancé en vue du mariage.

10 Famille nucléaire: un couple et ses enfants.

11 Famille monoparentale: Le géniteur ou la génitrice et son enfant. 
des pays du Maghreb et l'autre, d'origine européenne, en l'occurrence, la France (Kabbani, 1986: 4).

Historiquement, les clichés sur l'image des femmes orientales ont été largement conservés dans l'imaginaire collectif en Europe depuis le Moyen Âge. Ainsi, les femmes porteuses de la culture musulmane, ont eu, à l'époque, une double stigmatisation due, d'un côté, à sa condition de femme, en tant qu'êtres diaboliques et lubriques, considérées comme des ennemies de l'Eglise. C'est ainsi qu'elles ont subi une chasse aux sorcières, d'où les femmes étaient jugées par leur sexualité exacerbée, leur cannibalisme et leur association aux forces du mal. De l'autre côté, elles ont été stigmatisées en tant qu'Orientales, appartenant à l'État islamique; celui-ci tenu en compte comme l'ennemi menaçant non seulement l'Europe mais aussi la religion et la civilisation chrétienne. En d'autres termes, l'Orient était susceptible de préparer une offensive contre l'Occident à tous les niveaux voire, culturel, religieux, politique et militaire. Par conséquent, de cette époque médiévale ont perduré, jusqu'à nos jours, deux clichés fondamentaux sur l'image des femmes orientales (y compris celle de la femme d'origine maghrébine), à savoir, l'érotique et le religieux (Kabbani, 1986: 5).

Ainsi et c'est surtout à partir du XIX ${ }^{\mathrm{e}}$ siècle que l'image de la femme orientale est devenue une icône de la sensualité et de l'exubérance sans contestation, grâce aux différentes manifestations artistiques. Celles-ci issues d'une part de la Littérature, avec la traduction licencieuse des Mille et une nuits par Sir Richard Burton, où il décrit la rusée Shéhérazade, versée dans l'art de la persuasion, en racontant d'interminables histoires érotiques à son roi, en alimentant sa curiosité, afin de sauver sa peau. D'autre part, les peintres orientalistes, Eugène Delacroix, Luis Riccardo Falero et Jean Léon Gérôme ${ }^{12}$ entre autres, sont aussi responsables de répandre cette image d'érotisme associée aux femmes orientales en tant qu'objets sexuels, pervers et dévergondés, représentant de nombreuses scènes de femmes musulmanes languissantes sur des coussins, pratiquant leur toilettes dans les bains turcs ou dansant lascivement dans les cours royales, de telle sorte que le seul objectif de ces femmes consistaient à satisfaire les plus illicites désirs de leur mâles orientaux, puis ceux des européens en voyage. Or, la plupart de ces artistes, n'ayant pas voyagés aux pays de l'Orient, sont les responsables de la propagation de ce cliché érotisant, déjà considéré comme réel (Bury, 2010: 14).

Le deuxième stéréotype correspond à la version occidentale du statut des femmes dans l'Islam issu des récits des voyageurs du sexe masculin, aux XVII et XVIII ${ }^{\mathrm{e}}$ siècles. En fait, l'image des femmes musulmanes était celle d'une femme portant un voile, ignorante et soumise à la suprématie de l'homme que ce soit son père, frère ou mari. C'est ainsi que d'après la culture islamique, basée sur la religion, la femme était chassée de la vie publique, privée de toute instruction pour se consacrer exclusivement à la fonction procréatrice, aux

12 Pour plus de renseignements, consulter: <https://fr.muzeo.com/categorie/peinture/orientalisme> [14/06/2021]. 
soins de la famille, en particulier, l'obéissance aveugle aux besoins de son mari et des autres parents masculins. Cette situation d'inégalités subies par les femmes musulmanes sera prise comme prétexte européen pour "civiliser" l'Orient à travers la colonisation (Bilici, 2003: 22). Néanmoins, ce statut inférieur de la femme qui a connu sa pire version pendant la période de la Jahiliyya ${ }^{13}$, avant l'Islam, n'a pas été toujours présente dans la culture islamique. Après l'avènement de l'Islam, pour la première fois, on conféra, en théorie, aux femmes des droits égaux aux hommes dans tous les domaines, sauf celui de l'héritage. C'est grâce à l'encouragement du Prophète et de ses compagnons que la femme a eu accès, pendant le premier siècle de cette période, à tous les types de savoirs: religieux, artistiques, littéraires, médicaux, etc. Malheureusement, ce statut instruit des femmes s'est détérioré à cause d'une régression progressive des conditions économiques et sociales produite au Moyen Orient depuis le Néolithique. Dans son article cité antérieurement, Wijdan affirme que le développement de la vie urbaine, précipite la division du travail entre hommes et femmes, en exigeant le retour à celles-ci aux tâches domestiques, l'élevage des enfants les soins en générale du noyau familiale (Wijdan, 2002: 96).

C'est pourquoi le recul croissant subi par les femmes musulmanes, par rapport à leur rôle public dans la société, a été largement justifié par un détournement des hadits, en d'autres mots, les actes et les paroles dites par le Prophète Mahomet, en bénéfice de l'interprétation légale de la Sharia ${ }^{14}$ bien pratiqué par l'Islam fondamentaliste. Ainsi, cette situation d'infériorité par rapport à l'homme se succédera généralement tout au long des siècles, sauf dans quelques périodes, où leur statut était égal à celui de l'homme, par exemple, pendant les dynasties omeyyade, abbasside et fatimide (909-1171), ainsi qu'en Espagne musulmane (711-1494), aussi, suite à la Révolution française du 1789, grâce à l'inspiration des valeurs républicaines de liberté, égalité et fraternité (Hughes, 2013: 33).

Actuellement, et toujours selon Wijdan, le statut des femmes musulmanes (y compris la femme d'origine maghrébine en France) est plus varié et plus évolué par rapport à leurs ancêtres, grâce à l'accès à l'éducation et au développement des moyens de communication. Cependant, il ne faut pas identifier l'émancipation de ces femmes avec l'adoption d'un mode de vie tout à fait occidentale car elles sauvegardent des éléments propres de leur culture, voire, l'utilisation du voile pour des raisons diverses et non pas religieuses uniquement, mais pragmatiques, notamment, la protection contre le harcèlement sexuel et pour des raisons économiques (éviter d'aller chez le coiffeur, ni de s'habiller à la dernière mode). Également, le respect pour les fêtes religieuses, entre autres, constitue un signe d'identité qui ne les empêche pas de participer activement dans la société (Wijdan, 2002: 100-103).

13 Ou paganisme, c'est la période préislamique marquée par l'existence d'un panthéon d'idoles à la Mecque. Mahomet avait donné pour surnom à l'un de ses adversaires Quoriches le nom dégradant de Abû Jahl (père de l'ignorance).

14 Loi canonique islamique régissant la vie religieuse, politique, sociale et individuelle des musulmans. 
Du point de vue de l'apparence, les sociétés occidentales, y compris la française, se sont alimentées de préjugés contre toute notion de différence, provenant des stéréotypes choquants, spécialement ceux liés au look. Ces derniers sont les responsables de la stratification sociale en raison de cette apparence. En conséquence, les sujets sont rangés dans des catégories spécifiques, en les attribuant à des caractéristiques physiques, psychologiques et même intellectuelles. En réalité, l'image de la femme maghrébine en France obéit à la tyrannie des apparences, provoquant ainsi une véritable stigmatisation sociale, traduite, malheureusement, par une vision restreinte et stéréotypée d'ensemble, visant la discrimination de toutes ces femmes en raison à l'appartenance d'un groupe, celui de leur origine maghrébine sans accorder aucune considération à l'individualité de chaque sujet (Jabbour, 2013) ${ }^{15}$.

Par ailleurs, l'image de la femme d'origine maghrébine est typifiée d'après les standards de la société française, en relation à plusieurs paramètres: Tout d'abord, ses traits essentiellement méditerranéens, voire, sa peau tannée, ses yeux bruns maquillés de khol, et ses "[...] longs cheveux crépus, passés au henné, la femme sournoise, quémandeuse, voleuse peut-être" (Abouda, 2015: 140). Puis, son code vestimentaire, qui varie d'un côté, du plus classique pour l'immigrée de la première génération, selon Mozzo Counil, habillée à la façon traditionnelle, de ses robes longues, larges et colorées, sans oublier le port du foulard ou du hijab. Et de l'autre côté, au plus moderne et pragmatique du look occidental, pour celles de la deuxième génération, composé de pantalons, mini-jupes et même de décolletés "provocants", sans porter aucun accessoire de genre religieux (Mozzo Counil, 1994: 80-81). On trouve assez souvent ce contraste générationnel lié à l'apparence dans le quotidien occidental. Dans l'extrait suivant, l'enseignant bruxellois Frank Andriat décrit cette réalité que l'on pourrait aussi attribuer aux Français de souche:

[...] Ma mère et moi en rue à Bruxelles, elle la tête couverte d'un foulard, sa djellaba traînant sur le sol, moi avec une salopette rose bombons, un chemisier blanc et des chaussures à la mode. Cette dernière image, surtout, s'est gravée dans mon cœur. À elle est collée la remarque de deux garçons qui nous ont croisées ce jour-là. L'un dit à l'autre, en nous regardant ma mère et moi: - T'as vu quelle différence entre les deux générations! (Andriat, 2015: 113)

Ensuite, la question du prénom est aussi épineuse car elle renvoie à ces spécificités de la catégorie d'Arabe, présupposées par les Français de souche, de sorte que s'appeler Fatima, Aïcha ou Leila implique l'appartenance au monde musulman avec toutes les responsabilités que cela implique (Bouvet Maisonneuve, 2017: 84-86). Mais cela est plutôt propre aux femmes d'origine maghrébine de la première génération, tandis que

15 Thèse de doctorat présenté para Rhéa E. Jabbour. Pour plus de renseignements, consulter: <http://docplayer. fr/13839518-La-discrimination-a-raison-de-l-apparence-physique-lookisme-en-droit-du-travail-francais-etamericain-rhea-e-jabbour.html $>[14 / 06 / 2021]$. 
pour les beurettes, le choix d'un prénom arabe ou musulman préféré par leurs parents se fait, d'une part, pour marquer la distinction à l'égard des "vrais" Français, ceux de souche, et d'autre part pour affirmer la solidarité envers la communauté émigrée concernée. Par ailleurs, il est à préciser que la tendance à utiliser des prénoms considérés comme "mixtes" (Sonia, Sabrina, Sarah, Yasmine, etc...) constitue une façon de s'inscrire dans la société française tout en conservant le lien avec le milieu d'origine (Sicard, 2011: 70-71).

Enfin, les rôles typiques qui leurs sont accordés de façon implicite et quotidienne par la société de résidence, d'après les stéréotypes sur l'apparence, peuvent se résumer principalement en trois types, correspondant aussi à des qualités précises présupposées, en indiquant de plus, un niveau socio-économique déprimé: d'un côté, c'est le cas de la soumission de la gardienne des traditions, pour les immigrées de la première génération. De l'autre côté, c'est l'exotisme de la danseuse orientale, et l'analphabétisme de la racaille, pour les beurettes ${ }^{16}$. Ainsi, cette catégorisation basée sur des jugements, d'après l'apparence physique et des comportements, soi-disant relatifs à un groupe social, produit l'exclusion des femmes d'origine maghrébine du monde du travail, et par conséquent du développement du pays. Or, si ces femmes ont accès à l'activité professionnelle, grâce à leurs diplômes, elles rencontreront des difficultés pour s'y faire une place car elles seront également considérées, comme des femmes peu performantes et dynamiques, d'après les préjugés qui considèrent les femmes maghrébines en foulard comme des femmes au foyer (Barth \& Ramboarison-Lalao, 2013: 176-194). Au contraire, la prise en considération de ces femmes dans l'essor de la société s'accepte à part entière de celles provenant de la deuxième génération. Adila Benedjaï-Zou, cinéaste française d'origine algérienne, remet en cause les principes de la République française: "Pour les femmes de ma génération, la République française s'est montrée plus qu'accueillante. Elle offrait réussite social et reconnaissance, pour peu qu'on veuille bien épouser ses valeurs et rompre avec sa famille"17.

\subsection{La sexualité}

Le concept musulman d'une sexualité active chez les femmes est carrément différent à celui de la vision chrétienne, polarisée entre le bien et le mal, la chair et l'esprit, l'instinct et la raison. Ainsi, pour l'Islam, les instincts à l'état primitif sont énergie. Cette énergie provenant des instincts est considérée comme pure. Cela dit, la problématique du bien et du mal ne se pose pas dès le début, mais dans l'ordre social qui ordonne tous les aspects de la vie des individus. C'est pourquoi, d'ailleurs, le Coran réglemente à ce point la vie sociale, en faisant

16 Pour plus de renseignements, consulter: <http://www.lallab.org/top-8-des-stereotypes-de-femmes-raciseesdans-le-cinema-francais/> [14/06/2021].

17 Pour plus de renseignements, consulter: <https://www.lemonde.fr/culture/article/2019/06/20/aux-origines-descliches-sur-les-femmes-arabes-en-france_5479161_3246.html> [14/06/2021]. 
la séparation des sexes de façon stricte et réglementée, de telle façon que le comportement corporel des femmes est ainsi déterminé par un ensemble de lois et de rites rigides qui les protège en dépit de leur liberté, afin de respecter l'ordre musulman (Mernissi, 1983: 7-10). Ainsi, dès que la jeune fille devient adolescente, la mère l'instruit à cet égard en lui apprenant des consignes bien précises:

Maîtriser son corps déjà. [...] cacher ses cheveux qui sont un objet de désir, [...], maîtriser son enthousiasme, aussi bien que sa gourmandise [...] surtout ses élans de coquetterie, de provocation sensuelle, de sexualité. (Abouda, 2015: 139)

Conséquemment, les rencontres entre les hommes et les femmes ne se font qu'autour ces rites précis et codifiés sous le regard de la communauté, comme ceux du mariage, d'où la relation sexuelle n'est conçue que dans ses limites. C'est pour cela que la virginité se révèle comme une valeur fondamentale à maintenir pour la femme car, elle est liée à l'honneur de la famille. En effet, les jeunes filles sont instruites à cet égard, d'après toutes sortes de pratiques envisagées par la société, dans le but de la préserver. La plus connue de toutes ces pratiques est celle du rbat (action de nouer), connue aussi sous le nom de tezkar (action de fermer) ou encore tesfah (action de blinder). Par le biais de techniques ritualisées, cette pratique consiste en la fermeture symbolique de l'hymen avant la puberté et son ouverture, aussi symbolique, la veille du mariage. Ainsi, la chemise tachée de sang de la vierge après la nuit de noces représente la preuve indéniable de la sauvegarde de l'honneur familial. C'est "grâce" à ce type de croyances que la virginité en est devenue une véritable obsession. De nos jours, le certificat de virginité et la reconstruction de l'hymen par la chirurgie plastique viennent affermir ces pratiques traditionnelles du rbat (Ferhati, 2007: 174).

Il est vrai que les relations sexuelles hors du mariage ne sont conçues avec autant de transparence ni de publicité comme dans la culture occidentale, car elles sont considérées comme tabou. D'après le médecin psychiatre, Fatma Bouvet Maisonneuve, la sexualité en dehors du mariage non seulement existe aussi chez les jeunes femmes musulmanes, mais encore d'autres aspects qui y sont intimement liés, comme les maladies sexuellement transmissibles (MST) et les interruptions volontaires de grossesse desquels les instances médico-sociales prennent en charge, même si dans leur entourage familial, le non-dit reste la règle (Bouvet Maisonneuve, 2017: 58-59).

Dans cette ambiance d'interdits sociaux, les beurettes, ou descendantes des émigrés de la première génération, sont coincées entre les exigences de la tradition conservatrice, héritées de leur famille et un réel désir de liberté. En sorte que ces femmes en prennent position d'après deux attitudes opposées: L'une émancipatrice à travers la pratique de la sexualité avec des garçons européens, afin de se détacher des carcans de sa culture d'origine, vers l'intégration dans la société de résidence. Ainsi, elles doivent faire preuve d'une créativité performante pour mener à terme ses souhaits de se faire plaisir, en négociant avec la rigidité 
des codes sociaux, non sans éprouver de l'angoisse dans ses comportements sexuels. Et l'autre, protectrice en respectant les traditions de ses ancêtres aujourd'hui, dans une sorte de paradoxe ou évolution régressive (Bouvet Maisonneuve, 2017: 64).

En effet, la littérature se fait écho de cette problématique dans ces deux exemples, extraits de récits autobiographiques. Le premier, correspondant à l'écrivaine Aïcha Benaïsa, née en France de parents algériens, où elle raconte ses soucis à ce sujet, lors d'une rencontre avec Antonio:

[...] Antonio a été le premier. Pas un premier comme tout le monde en a eu. J'avais dixneuf ans et toutes les amies "l'avaient déjà fait". Mais pour moi, c'était quelque chose de très important, de très grave. Antonio s'est rendu compte de l'importance qu'avait pour moi la virginité. Il ne connaissait rien à l'Islam. Je lui ai expliqué, j'ai voulu lui faire partager un peu de ma culture, pour qu'il sache bien avec qui il était... Je lui ai dit que je n'avais jamais eu de rapports sexuels, que je ne savais pas si j'en aurais avec lui. Il a très bien réagi, en m'expliquant qu'il était bien avec moi quoi qu'il arrive. Il n'a pas insisté et n'en a pas reparlé. J'étais rassurée. C'était exactement le contraire de ce que ma mère m'avait dit sur les hommes. (Benaïssa, 2015: 135)

Le second, celui de la cinéaste et fondatrice du groupe musicale Djurdjura, Djura Abouda explique sa rencontre libératrice et prochaine fuite avec Olivier, et surtout les normes à respecter par les femmes issues du code social, concernant la virginité, en outre, des conséquences de pas obéir les traditions:

[...] Il se montra d'une attention et d'une patience infinies, acceptant nos rendez-vous furtifs, dans les cafés, ici et là, ou plus souvent dans sa voiture, pas trop loin de chez moi pour que je puisse rentrer vite. Il dut se plier aussi à ma pudeur: nous vivions un amour on ne peut plus platonique. Je restais marquée, malgré tout, par les principes ancestraux: on ne fréquente pas un garçon avant le mariage. Je me croyais émancipée: j'abordais cette rencontre intime avec le poids de tous les tabous. J'allais perdre ma virginité hors les liens du mariage. J'imagine à quel point cette réaction peut paraître anachronique, mais c'était ainsi. Le contenu symbolique de la virginité poursuit chez nous la jeune fille dès qu'elle se trouve pubère. Le lendemain des noces, les draps tachés de sang virginal sont exposés à la fenêtre, prouvant à tous la vertu de la nouvelle épouse. Quant à se rendre coupable d'actes sexuels avant la cérémonie nuptiale, on m'avait assez expliqué, à Ifigha, que c'était sanctionné du châtiment suprême, même si la fille avait été violée! (Abouda, 2015: 140)

Au-delàs de son audace et sa bravoure pour aborder le sujet, presque interdit, de la sexualité féminine, Mahfoudh Draoui (2016) nous exhorte à ne pas oublier l'incontournable contribution pour l'émancipation des femmes Arabes que l'écrivaine marocaine, Fatima Mernissi, a réalisée tout au long de sa carrière, en particulier, dans les domaines de la scolarisation, le travail salarié et la participation politique. 
La bande dessinée, Hshouma ${ }^{18}$ de la jeune caricaturiste marocaine, Zainab Fasiki (2019), aborde ce sujet tellement délicat de la sexualité féminine, ayant pour but de porter plainte contre tous les taboues qui entourent le corps de la femme ${ }^{19}$. C'est ainsi que lors du séminaire sur le féminisme dans le monde arabe, organisé par les professeures Eva Lapiedra et Naima Benaicha en mars 2019, que la jeune artiste-dessinatrice marocaine a fait part de sa lutte pour instruire les femmes maghrébines à cet égard, moyennant ses illustrations et ses fanzines, pour réussir leur affranchissement en dépit du harcèlement et de la censure dont elle a toujours été objet dans son pays d'origine.

\subsection{L'Éducation nationale pour l'apprentissage des valeurs républicaines}

Le système éducatif français sert à véhiculer les éléments fondamentaux du fonctionnement de la République. Ainsi, dans les établissements scolaires, comprenant l'enseignement primaire et moyen (l'école et le collège) et secondaire (le lycée), on forme les élèves en matière d'éducation civique, politique et juridique, afin de créer des générations actives et engagées envers ses congénères tout en ayant un sentiment profond d'appartenance à la nation. C'est pour cela que dans chaque centre éducatif, l'on enseigne l'importance capitale que la Révolution française de 1789 a fait dans le changement du paradigme politique de la France, en éliminant la monarchie absolue pour embrasser le nouveau régime républicain, constitué comme démocratique, c'est-à-dire, le pouvoir appartient au peuple et c'est lui qui élit les députés et le Président de la République par suffrage universel direct. De même, elle s'érige comme sociale, puisque l'Etat cherche à réduire les inégalités et à assurer la solidarité grâce à la Sécurité Sociale (Costa Lascoux, 1991). Les éléments qui composent la notion de République française ${ }^{20}$ sont comme suit: le texte fondateur se trouve dans La Déclaration des Droits de l'Homme et du citoyen de 1789; les valeurs à atteindre dans sa devise, voire: Liberté, Egalité et Fraternité; les principes, comme des règles d'action pour mettre en œuvres ces valeurs, tels que l'indivisibilité de la nation, la laïcité, et l'esprit démocratique et sociale et les symboles ${ }^{21}$ : le drapeau tricolore, l'hymne de la Marseillaise, la Marianne et son bonnet phrygien, comme emblème de la liberté, la fête nationale du 14 du juillet, le coq tricolore pour les rencontres sportifs ainsi que la langue française comme signe identitaire. Depuis 1792

18 Ou Hshouma: Corps et sexualité au Maroc, signifie "honte", en arabe dialectal maghrébin. Ce terme est lié à l'honneur qui pèse sur les épaules de la femme. Dans les familles traditionnelles conservatrices, aussi bien chrétienne que musulmanes ou judaíques, l'honneur est sauvé ou perdu grâce ou à cause de la femme. Voir aussi, Au delàs de la pudeur de Soumaya Naamane-Guessous.

19 Pour plus de renseignements, consulter: <https://smoda.elpais.com/feminismo/zainab-fasiki-como-derrocar-al-patriarcado-marroqui-con-un-simple-lapiz/> [03/06/2021].

20 Pour plus de renseignements, consulter: <https://www.education.gouv.fr/les-valeurs-de-la-republique-1ecole-1109> [03/06/2021].

21 Pour plus de renseignements, consulter: $<$ https://www.gouvernement.fr/charte/charte-graphique-les-fondamentaux/les-symboles-de-la-republique-francaise $>$ [07/06/2021]. 
et en relation avec l'idée d'adaptation face à la réalité de l'esprit français, la France a eu cinq républiques avec une différence sur la répartition et les fonctions du pouvoir exécutif et législatif. À partir de la cinquième, ce système politique devient plus stable et ce grâce à la fixation des principes de la République française dans la Constitution du 4 octobre de 1958. Les Présidents qui ont été en tête de l'Hexagone depuis cette date sont: Charles De Gaulle, Georges Pompidou, Valéry Giscard d'Estaing, François Mitterrand, Jacques Chirac, Nicolas Sarkozy, François Hollande et actuellement, Emmanuel Macron, qui ont militaient dans différentes idéologies, de droite ou de gauche, mais toujours dans une position “mitigée”, c'est-à- dire ni dans l'extrême droite, ni dans le communisme. Il faut préciser que ces éléments clé de la République française vont de pair avec les grands principes du système éducatif français ${ }^{22}$, voire, la liberté de l'enseignement, la gratuité, la laïcité, la neutralité et l'obligation scolaire.

La considération de la langue arabe n'a pas l'importance qu'elle mérite dans ce cadre scolaire, même si elle représente la deuxième langue parlée en France, en raison de la prépondérance de l'immigration maghrébine. En effet, l'enseignement de l'arabe en France semble peu à peu échapper à l'institution scolaire, il risque, même, d'occuper une place symbolique, malgré les liens historiques entre les deux cultures, voire, la française et la maghrébine. De cette manière, la langue arabe occupe une place peu soulignée dans la gestion, dans l'enseignement des langues étrangères, dans les établissements publiques (les collèges et les lycées), etc. (Cheikh, 2010: 95-96). L'arabe ainsi est, ainsi, rarement proposé aux élèves comme deuxième langue, en échappant même, aux recommandations des recteurs et de l'inspection académique. Cette dispensation de l'Éducation Nationale par rapport à l'étude de la langue arabe se traduit en sa suppression du programme de CAPES ${ }^{23}$, fermeture programmée de classes d'arabe, régression du nombre de professeurs, baisse constante du nombre d'élèves, etc. C'est ainsi que les associations culturelles et $1^{\prime} E L C O^{24}$ essayent de répondre à la demande et de combler ce vide laissé par les établissements scolaires, en offrant une éducation mixte, comprenant des cours de langue et d'éducation islamique, en considérant ainsi l'incontournable aspect religieux de la culture maghrébine ${ }^{25}$.

Parmi les possibles raisons de ce désengagement de l'institution scolaire, on trouve le statut de l'arabe comme langue de minorités et d'immigration, l'intérêt des élèves pour les autres langues proposées à l'école publique, la concurrence des écoles communautaires de tout genre, qui ont plus d'attrait pour le public concerné, essentiellement issu de l'immigration maghrébine et enfin, l'image de l'Islam et des Arabes dans le monde (Cheikh, 2010). Concernant ce dernier aspect, c'est le rapport de la langue arabe à la religion musulmane

22 Pour plus de renseignements, consulter: <https://www.education.gouv.fr/les-grands-principes-du-systeme-educatif-9842> [08/06/2021].

23 CAPES: Certificat d'Aptitude au Professorat de l'Enseignement du Second degré.

24 ELCO: Enseignement des langues et de culture d'origine.

25 Pour plus de renseignements, consulter: <https://www.lemonde.fr/idees/article/2014/02/11/il-faut-enseigner-1arabe-dans-le-service-public_4364171_3232.html $>$ [07/06/2021]. 
qui se révèle opposé aux valeurs de laïcité de la République française ne permettant aucune manifestation religieuse, ou bien, lié de façon erronée et systématique au radicalisme. Cependant, Bouvet Maisonneuve manifeste:

\begin{abstract}
Nous ne pouvons pas d'un côté renforcer et souligner l'importance de la laïcité dans notre pays et dans l'enseignement et ne pas proposer l'enseignement de la langue et de la civilisation arabes dans le cadre de l'éducation nationale, c'est-à-dire un cadre laïc. Le risque est de continuer de laisser filer vers les mosquées ceux qui sont en demande. (Bouvet Maisonneuve, 2017: 108)
\end{abstract}

Le problème, selon elle, réside dans le fait d'apprendre scrupuleusement l'arabe dans le cadre religieux, à l'écart des autres valeurs civiques, sociales, culturels, etc. aidant à développer l'esprit critique des jeunes. Certes, cela peut induire à des interprétations fragmentaires de la part de "prédicateurs de pacotille", d'où, les mosquées et les écoles coraniques sont des endroits susceptibles d'endoctrinement vers le radicalisme, dont le fléau est le terrorisme islamique.

Néanmoins, l'étude de la langue arabe, selon la psychiatre Bouvet Maisonneuve, ne concerne que les Français d'origine arabe puisqu'elle est diffusée dans le cadre de l'enseignement supérieur, notamment à l'Inalco ${ }^{26}$, en raison de l'importance de cette langue dans le domaine international des affaires.

Par ailleurs, il est indispensable de ne pas ignorer le statut des élèves et des étudiantes Françaises d'origine maghrébine, car l'Éducation nationale les a mis dans l'oxymore d'exceptionnelles ratées qui englobe l'ensemble de ce groupe social sans valoriser les individualités de chaque sujet. C'est-à-dire, soit elles sont considérées comme des exemples hors norme par leur intelligence, soit elles appartiennent à la catégorie de cas insurmontables, dû à leur manque de compétences. Parfois, ce sont les propres membres de cette communauté qui se trouvent prisonniers dans ces clichés, en contestant le statut d'un sujet concret. Voici un exemple tiré de la littérature autobiographique. Il s'agit d'une réflexion critique sur le traitement des lycéennes, plus précisément de la jeune fille Anne, avant nommé Sélima, à l'égard des élèves maghrébines:

[...] On nous a injecté de l'exception à haute dose... Moi, je suis un exemple vivant! [...] Et si j'en avais marre de représenter à moi toute seule les immigrées? Et si j'en avais marre de servir de caution? [...] Combien il y en a des filles algériennes et mêmes des Arabes [...] et même des immigrés qui passent le bac cette année en France. (Feraud, 2015: 42)

26 L'Institut national des langues et civilisations orientales, dit Langues O', est un établissement français d'enseignement supérieur et de recherche chargé d'enseigner les langues et civilisations autres que celles originaires d'Europe occidentale. 
Le cas des jeunes filles scolarisées répond aux attentes d'ordre intégrationniste de la société française, puisque celle-ci fait de la fille scolarisée et "bonne élève", le vecteur et la figure emblématique du projet assimilationniste de l'école et de la république. Or, ces filles ne trouvent aucune place dans l'Éducation nationale française pour approfondir sur leur culture d'origine, la maghrébine. D'où leur sentiment d'humiliation, d'abandon, et de mépris, visant les problèmes sur l'affirmation d'une identité partagée en deux: l'une, soi-disant, française en attendant l'adhésion aux valeurs et principes républicains dont elles se sentent exclues. Et l'autre, l'arabe avec l'ambiguïté que les Arabes de France éprouvent à l'égard de "leur" langue, conçue comme élément d'identification plus que de communication (Qribi, 2017: 3). Résultat de courses, ce vide dans le cadre scolaire officiel favorise l'apprentissage de la langue arabe soit dans les écoles des associations pour les immigrés, soit dans les écoles coraniques ou dans les mosquées, pouvant entraîner un repli des Français d'origine maghrébine vers le communautarisme tellement critiqué par le gouvernement français dans ses politiques d'intégration, aggravant encore davantage cette brèche sociale qui fait distinguer deux catégories de citoyens parmi les Français: ceux de première classe, voire les Français de souche, et ceux de la deuxième classe, appartenant à la communauté arabe. De même, cette distinction sociale comporte la remise en question de la devise républicaine: Liberté, Égalité, Fraternité, si bien que la favorisation de situations de vulnérabilité, d'injustice et de racisme spécialement pour les femmes d'origine maghrébine, en attendant leur condition de genre et leur appartenance à une minorité ethnique dans ce pays considéré par le groupe de Hip Hop français d'origine tunisien Puzzle ${ }^{27}$ comme "La France Fiction, où Liberté est un mot encore en construction dans le pays des droits de l'Homme". Nonobstant, l'apprentissage de l'arabe est apprécié et favorisé dans l'enseignement supérieur, donc, non obligatoire, selon des intérêts strictement économiques et commerciales, car c'est dans les relations internationales que les arabes occupent une place privilégiée en devant de l'autre communauté la plus nombreuse au monde: la chinoise. ${ }^{28}$

En attendant de résoudre cette omission flagrante et manifeste de l'arabe dans le contexte académique obligatoire, il est évident que la communauté maghrébine, en France, n'arrive pas à se définir de manière harmonique pour embrasser les principes républicains sans heurter son identité, puisque le gouvernement français n'accorde pas à la culture de base, voire la langue arabe, la place qu'elle mérite. Également, les clichés polarisés sur les compétences intellectuels des femmes d'origine maghrébine, compris comme des exceptions autant positives que négatives dans la norme, ne favorisent pas non plus leur adaptation dans la couche académique du pays.

27 "France fiction" on vient m'chercher (2006) CD, France, Discographie.

28 Pour plus de renseignements, consulter: $<$ https://francais.rt.com/inernational/4255-oublies-francais-chinois-petits-britanniques $>[06 / 06 / 2021]$. 


\subsection{La religion musulmane en France: Islamisme vs laïcité}

La question religieuse est l'autre grand sujet de la culture arabe qui a été aussi négligé par la France. À cet égard, aussi bien le gouvernement français que la communauté musulmane doivent se mettre d'accord pour mener à bien le "vivre ensemble". Ainsi, d'un côté, la société française doit reformuler ses notions de nation et de laïcité pour garantir la diversité culturelle, en octroyant une place représentative à l'Islam, en tant que la deuxième religion de l'Hexagone ${ }^{29}$ et ce en dépit des critiques, parfois excessives et fondées, dans le débat sur l'intégration (Lamchichi, 2002). C'est ainsi que la nation française repose sur la conception abstraite d'État qui ne reconnaît que des citoyens libres et égaux en droits et dignité, sans prendre en considération les diverses particularités des communautés soit ethniques, culturelles, linguistiques ou religieuses dans la vie publique, en faveur du respect des valeurs républicaines dont la protection du cadre laïque et le rôle de l'école publique sont fondamentaux dans la socialisation. Nonobstant, l'appartenance religieuse n'est pas assaillie par l'État, puisque celui-ci lui confère une totale liberté dans la sphère privée, c'est-à-dire seulement si elle n'a pas la vocation de transgresser les lois républicaines au sein de la laïcité. D'autre part, les musulmans doivent contribuer à renouer leur vision différenciatrice d'identification avec la religion, associée au communautarisme vers l'ouverture et la participation active dans la vie démocratique de la société d'accueil. En conséquence, le but, qui s'avère complexe, est celui de trouver un équilibre entre le respect de la laïcité et l'exercice de la liberté religieuse, visant à éviter l'exclusion sociale (Ragi, 2002: 7).

Cependant, il faut tenir en compte la relation des femmes musulmanes d'origine maghrébine avec l'islam, car il peut y avoir deux selon Frédérique Sicard: l'appartenance religieuse ou identitaire. La première est basée sur la pratique religieuse, à laquelle s'accrochent celles issues de la première génération d'immigrées, en qualité de gardienne des traditions. Ainsi, leur pratique est basée sur le respect des dogmes et des normes spécifiques telles que le jeûne, la fréquentation de la mosquée, les interdits alimentaires (halal vs haram) ${ }^{30}$, ainsi que les manifestations des fêtes religieuses comme L'Ä̈d el-Saghir ${ }^{31}$, ou "petite fête" qui clôt le mois de jeûne du Ramadan"32; ou l'Aïd el-Kébir "33،la grande fête" où l'on sacrifie le mouton en commémoration du dévouement d'Abraham mentionné dans le Coran comme dans la Bible. Quant à la deuxième, c'est par affiliation culturelle et identitaire et non de croyance que les Françaises musulmanes (beurettes) se considèrent en tant que tels (Sicard, 2011).

29 Pour plus de renseignements, consulter: <https:/www.vie-publique.fr/rapport/24979-lislam-dans-la-republique $>[18 / 06 / 2021]$.

30 Halal, licite par opposition à haram, illicite.

31 Il s'agit de l'Aïd al-fitr ('Īd al-Fițr) en arabe littéraire et dans le reste du monde arabe.

32 Pour plus de renseignements, consulter: <https://www.terrafemina.com/societe/societe/articles/28080-quest-ce-que-le-ramadan-origine-signification-et-traditions.html > [06/06/2021].

33 Il s'agit de l'Aïd al-adha ('Īd al- al-'aḍhā) en arabe littéraire et dans le reste du monde arabe. 
Alors, il n'est pas correcte de comparer toutes les femmes Maghrébines de France comme étant des femmes musulmanes pratiquantes, surtout, parce qu'un nombre important de ces femmes, appartenant à la deuxième et troisième générations comprennent leur émancipation comme individus selon la vision laïque de la société française, donc à l'écart de la religion et de l'utilisation des symboles caractéristiques comme le hijab, qui impliquent la catégorisation dans les stéréotypes discriminatoires abordés, tout en gardant ces attaches identitaires propres de leur culture d'origine (Badinter, 2005).

\subsection{Le racisme}

La France prend une position contradictoire à cet égard, puisque d'un côté, les Français s'affirment comme de plus en plus tolérants envers les minorités ethniques, linguistiques et culturelles, y compris la maghrébine, selon le rapport annuel de la Commission Nationale Consultative des Droits de l'Homme $(\mathrm{CNCDH})^{34}$. Or, la tolérance varie peu depuis 2016 et oscille autour de 64 points face aux 66 points en 2019, sur une échelle de 0 à 100 . D'après ce rapport, les discriminations et les actes racistes persistent au jour le jour, de manière indirecte parfois sous des formes subtiles difficiles à caractériser et à dénoncer pour les personnes qui en sont victimes. Ainsi “[...] les vieux stéréotypes décrétant l'infériorité physique et morale des minorités ciblées, font place à des pseudo-arguments culturels destinés à établir une incompétence entre les valeurs et les mœurs de certaines 'races' avec la République". ${ }^{35}$

34 Pour plus de renseignements sur ce rapport, consulter: <https://www.cncdh.fr/fr/publications/la-cncdh-presente-le-30e-rapport-sur-la-lutte-contre-le-racisme-lantisemitisme-et-la $>$ [12/06/2021].

35 Pour plus de renseignements, consulter: <https://www.lemonde.fr/societe/article/2020/06/18/stereotypes-discriminations-et-actes-racistes-ne-reculent-pas-en-france-selon-un-rapport_6043291_3224.html> [08/06/2021]. 


\section{ZOOM SUR LES CHIFFRES DU SCRT}

Le SCRT est un outil opérationnel permettant de mesurer à un instant T les actes racistes subis par les personnes. Les chiffres du SCRT se fondent sur les remontées provenant de ses relais territoriaux, ses partenaires locaux, des médias ainsi que des associations représentant les communautés religieuses musulmane et juive, avec lesquelles il a un partenariat. Ces chiffres ne sont donc pas exhaustifs et comportent un certain nombre de biais.

\section{EVOLUTION GLOBALE DES FAITS RACISTES COMPTABILISÉS PAR LE SCRT SUR LE LONG TERME}

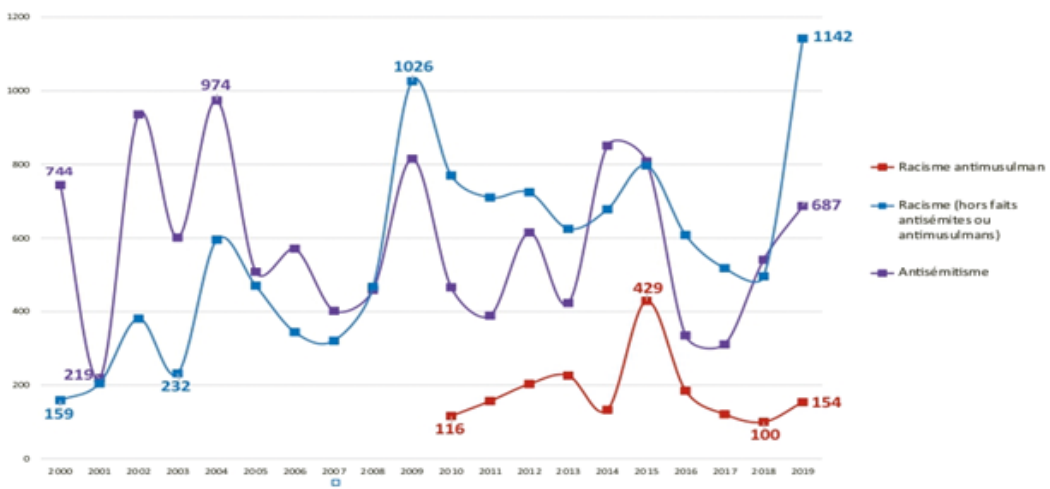

EVOLUTION DES FAITS RACISTES ENTRE 2018 ET 2019

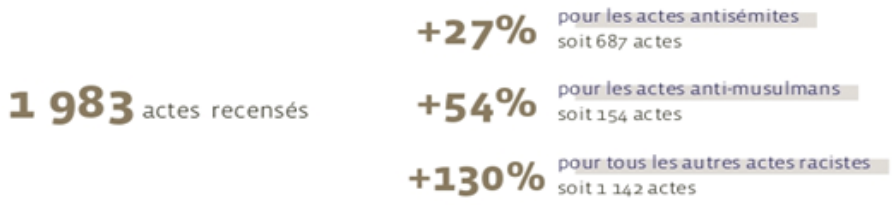

Source: Rapport sur la lutte contre le racisme, l'antisémitisme et la xénophobie (2019).

À ce sujet, les femmes d'origine maghrébine constituent une cible largement représentative dans la société française, en outre de sa condition de genre tellement dévalorisée dans le système capitaliste et patriarcal que partagent toutes les femmes européennes, aussi bien les Françaises de souche que le reste d'étrangères. Dans ce sens, le Service de Recherche du 
Parlement Européen $\left(\mathrm{EPRS}^{36}\right)$ reconnaît que malgré les progrès considérables des dernières années, la situation entre les femmes et les hommes reste, toujours, inégale dans l'Union Européenne, notamment dans les domaines de l'emploi, les ressources économiques et financières, le leadership, le temps consacré aux responsabilités familiales, la violence conjugale subie para les femmes, etc.

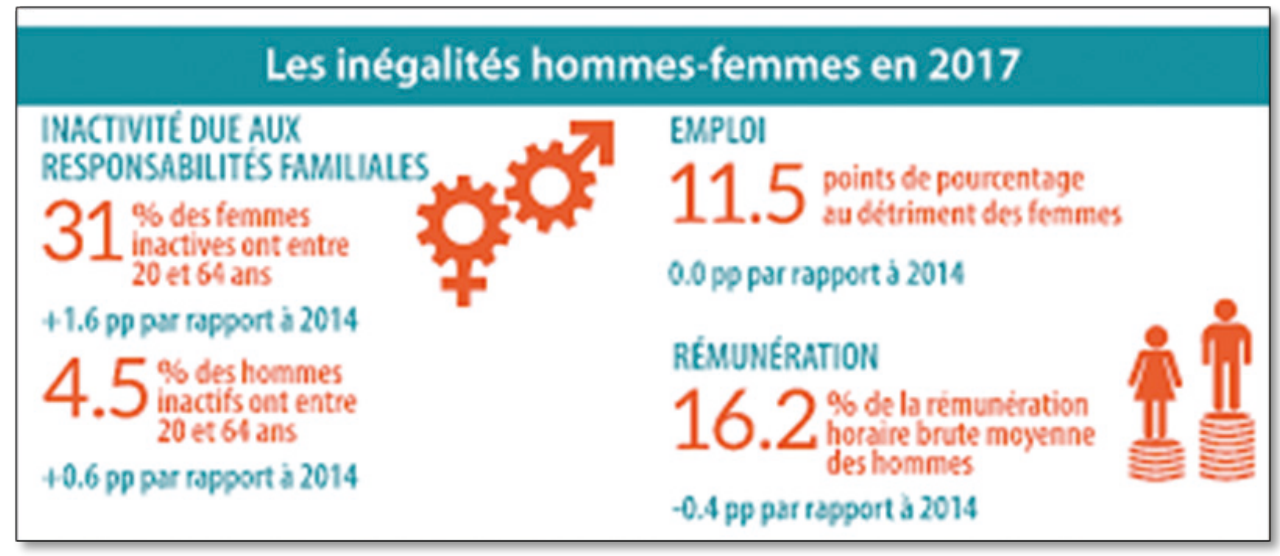

Source: Promouvoir l'égalité entre les femmes et les hommes.

Tahar Ben Jelloun, quant à lui, affirme que le rejet de l'étranger, du différent, de celui qui est considéré comme une menace pour la sécurité sociale est un réflexe universel et n'épargne aucune société. Ainsi, une fois c'est le juif qu'on persécute, une autre c'est le Noir ou l'Arabe et selon l'époque et le lieu où l'on se trouve, c'est aussi le Blanc qui est visé et méprisé. L'écrivain d'origine marocaine, établi en France depuis les années 70, auteur du roman Le Racisme expliqué à ma fille propose la démarche pédagogique dans les écoles comme une issue pour démonter les mécanismes et l'absurdité des bases de ce fléau, à son avis, issu de la peur et l'ignorance, tout en restant vigilant afin de faire de la lutte contre le racisme une habitude du quotidien (Ben Jelloun, 1998: 62).

Quant à Christelle Hamel, celle-ci considère que les femmes d'origine maghrébine subissent le racisme, qui loin de se limiter au discours de l'inégalité des races, consiste en l'application d'un ensemble de pratiques discriminatoires dans l'accès au travail, au logement, aux loisirs et à l'activité politique. Parmi ces aspects, celui du monde du travail semble la plus pertinente à commenter dans cette étude (Hamel, 2006). Cela dit, l'évolution des carrières des femmes d'origine maghrébine en France varie en fonction des conditions d'arrivée et de séjour, de motivations, des origines sociales et des spécificités culturelles. On est donc face à un groupe hétérogène des femmes compris par plusieurs profils professionnels.

36 En anglais: European Parliament Reasearch Service. 
Malheureusement, celles-ci constituent la proie des stéréotypes imposés par la discrimination, quelques soient leurs particularités. De même, les principales difficultés rencontrées par ces femmes concernent leur insertion dans le marché du travail et ce quel que soit leur niveau de qualification. D'après Zeineb Touati, il y a deux profils spécialement sensibles: le premier appartient aux femmes immigrées de la première génération arrivées dans les années 70, dans le cadre du regroupement familial. Celui-ci est représente le cliché de l'image de la Maghrébine mère de famille nombreuse, soumise et inactive qui reste loin d'être incluse dans le monde du travail. Elles sont généralement analphabètes et maîtrisent très peu la langue française. En plus, elles viennent d'un milieu social où elles ne doivent pas et ne peuvent pas travailler. Cependant, ces femmes sont parfois obligées de travailler en raison de besoins économiques, en faisant face aux difficultés issues du choc culturel qui suppose s'adapter au marché de travail français. C'est à elles que l'on attribue les travaux les plus pénibles et les moins rémunérés, dû à leur manque de formation. Quant au deuxième profil, celui-ci correspondant aux beurettes nées en France de mères de la première génération. Celles-là subissent la discrimination par rapport à l'embauche et aux rémunérations en raison de leur origine maghrébine, bien qu'elles possèdent des diplômes et de l'expérience professionnelle propres du poste de travail requis. Le racisme joue certainement un rôle important, comme en témoigne le nombre croissant de plaintes portées pour discrimination à l'emploi, ou encore les statistiques données par des organisations reconnues comme SOS Racisme (Touati, 2012). De même, il ne faut pas négliger la discrimination chez les femmes d'origine maghrébine concernant la place et la considération qu'elles occupent dans la société française par rapport au reste de femmes étrangères, en particulier, celles d'origine brésilienne qui jouit de la sympathie spontanée des Français de souche. C'est le cas du personnage cinématographique, Walid, d'origine algérienne qui avoue à son ami Samba (d'origine sénégalais) sa performance identitaire en Wilson, car, il s'était rendu compte, dès son arrivée à Paris, que grâce à la colocation avec des brésiliens: “pour le boulot, pour les meufs, c'est plus facile d'être Brésilien qu'Algérien [...]"37

\subsection{Les modèles politiques d'intégration: Assimilation vs multiculturalisme}

Au début des années 2000, l'Union Européenne conçoit la question de l'intégration des femmes immigrées comme un enjeu social et politique de premier ordre, à la suite de programmes européens et internationaux, ayant pour but leur promotion et leur protection dans le monde. En France, Le Haut Conseil à l'Intégration (HCI) prend position à cet égard, moyennant des accords-cadres ${ }^{38}$ signés en 2003 et renouvelés en 2007, relatifs aux femmes

37 Pour plus de renseignements, consulter: Nakache, Olivier \& Toledano Enric, Samba, France, fiction, 115 minutes, 2014.

38 Ces accords-cadres sont signés par le Service des droits des femmes et de l'égalité, la Délégation interministérielle à la Ville (DIV), la Délégation générale à l'emploi et à la formation professionnelle (DGEFP), l'Agence 
immigrées maghrébines, ou issues de l'immigration maghrébine, pour favoriser leur parcours d'intégration et prévenir et lutter contre les discriminations (Kulakowsky, 2008). Toutefois, la question de l'intégration de ce groupe de femmes dans la société française continue à susciter des conflits, puisque, malgré ces politiques menées par le gouvernement, les données sont floues et même opposées. D'une part, le discours politique des agents de l'État dément qu'il existe des problèmes spécifiques d'intégration pour les femmes issues du Maghreb, que ce soit pour celles de la première génération, ou pour celles nées en France, descendantes de celles-ci. Ils font, même, preuve du contraire, en essayant de prouver qu'elles se fusionnent sans grands efforts dans la société française. D'autre part, l'opinion publique se préoccupe du supposé repli communautariste en raison de leurs revendications pas satisfaites, concernant leur spécificité culturelle maghrébine (Gourdeau, 2015). De plus, la question des femmes immigrées est intimement liée à celles du chômage, du racisme, du sexisme, de l'éducation, et de la religion, avec tous les problèmes, que cela implique, notamment, ceux attachés aux stéréotypes, comme les principaux responsables de leur manque d'adaptation (Vasseberg, 1997). Quoi qu'il en soit, l'acclimatation des femmes d'origine maghrébine dans la société française, semble un véritable défi, (étant donné les modèles politiques d'intégration disponibles), car, d'un côté, l'assimilation adhérant le concept de citoyen français, dégagé de toute particularité ethnique, heurte nécessairement leur culture d'origine, surtout, dans deux de ses éléments, les plus importants: l'un, la religion musulmane qui ne trouve aucune place reconnue, dans l'ensemble institutionnel français, en raison du respect de la laïcité et l'autre, la langue arabe, pas suffisamment promotionnée dans l'enseignement obligatoire (Constantina, 2012: 575).

De l'autre côté, le multiculturalisme français proposé est loin de mettre en pratique la politique multiculturelle britannique, sous la forme de droits collectifs, dans la concession des espaces publiques aux minorités, pour permettre une cohabitation agréable entre les divers groupes ethniques et religieux, dans une société politique libérale et tolérante. $\mathrm{La}$ France donc, accorde le développement de ces particularités culturelles maghrébines, mais, uniquement dans la sphère privée, en favorisant ainsi la rupture sociale entre les immigrées maghrébines et la population autochtone. Néanmoins, le gouvernement français utilise en son bénéfice, ce supposé multiculturalisme actif, afin de prouver à l'opinion publique, son esprit solidaire, inclusif et équitable, contenu dans sa devise. C'est donc à travers la conception "Blanc, Black, Beur" 39 , issue de la victoire de l'équipe de France de football, dans la coupe du monde en 1998, que la nation française veut exporter au monde, en tant que pays où le "vivre ensemble" ou la cohabitation en totale harmonie parmi les diverses cultures est une réalité plausible.

pour la cohésion sociale et l'égalité des chances (ACSE) et l'Office Français d'immigration et d'intégration (OFII).

39 Pour plus de renseignements, consulter: <https://www.liberation.fr/debats/2018/07/17/black-blanc-beur-la-find-une-generation_1667033>[13/06/2021]. 


\section{Conclusion}

Cette étude nous a permis de confirmer que l'identité française semble être encore une question polémique. Notre objectif à travers ce travail a été de rassembler le maximum de références bibliographiques sur un sujet qui cerne l'ambigüité identitaire spécifique à la femme issue de 1'immigration maghrébine. Par conséquent, l'État français devrait (re)penser sa notion restreinte de citoyen français, pour ainsi prendre en compte les particularités des diverses cultures qui la composent, visant, ainsi, une véritable société multiculturelle, où le respect, la tolérance et le partage soient la garantie contre toute sorte de communautarisme. En vue de l'éradication des inégalités, la France devrait dorénavant s'adhérer à la notion de "défaire le genre", à savoir, l'anéantissement des catégories traditionnelles par rapport au sexe de l'homme et de la femme, responsables de l'assujettissement de celle-ci sous la domination masculine. Par ailleurs, la diversité culturelle devrait être comprise comme une richesse et non comme une menace, puisque les différences, loin de servir comme justificatif pour inciter aux conflits, elles peuvent être, plutôt, prises en considération pour la création d'espaces de rencontre, dans l'intérêt du dialogue et de l'entente mutuelle. François Jullien (2021) témoigne que les vis-à-vis des cultures ne devraient pas conduire à penser le monde de la diversité culturelle comme une juxtaposition d'altérités où chacune se replie sur soimême, car c'est précisément l'écart qui fait tenir l'ensemble et créer la cohérence. De cette manière, la catégorie de l'altérité sort de l'immobilisme de l'essence pour se révéler comme outil de dialogue.

Á notre avis, la France mutile négligemment une partie inhérente de son identité, car en refusant la multiculturalité et l'interculturalité, elle se prive volontairement de ce dialogue vif et enrichissant pour son auto-connaissance intégrale et cela passe non seulement pour la reconnaissance de ses charités, mais encore pour l'illumination de ses propres ténèbres, puisque, en fin de compte, "l'autre" n'est que le reflet de soi-même dans le miroir de ses propres contradictions.

Pour aboutir à cela, l'Hexagone devra, nécessairement, assumer, accepter et surtout embrasser chacune de ces spécificités culturelles, y compris la maghrébine, comme partie inhérente de son identité multiple et pleine de contrastes. Seulement de cette façon, la France deviendra la digne méritante de sa devise: Liberté, égalité et fraternité. Ainsi on pourra conclure en rappelant ce que Mona Ozouf a remarquablement résumé: “Être français est sans doute une décision, mais aussi un héritage" 40 .

40 Pour plus de renseignements, consulter: https://www.lepoint.fr/societe/mona-ozouf-pourquoi-nous-n-arrivonspas-a-faire-cohabiter-nos-multiples-identites-13-01-2011-129512_23.php\#> [12/05/2021]. 
Anales de Filología Francesa, n. ${ }^{\circ}$ 29, 2021

NAIMA BENAICHA ZIANI Y AZUCENA ORTIZ GARCÍA

\section{Références bibliographiques}

Aвouda, Djura. 2015. "Le voile du silence" in Luc Collès (éd.). L’immigration maghrébine dans la littérature française, anthologie France-Belgique (1953-2010). Bruxelles, EME éditions, 140-170.

ANDRIAT, Frank. 2015. "Le journal de Jamila" in Luc Collès (éd.) L'immigration maghrébine dans la littérature française, anthologie France-Belgique (1953-2010). Bruxelles, EME éditions, 113-114.

BADINTER, Élisabeth. 2005. "La laïcité, un enjeu pour les femmes" in Matériaux pour l'histoire de notre temps, 78, 50-53.

BARTh, Isabelle \& Ramboarison-Lalao, Lovanirina. 2013. "Perception de l'apparence dans le monde du travail: le poids des préjugés. Enquête auprès de 909 postiers" in Management \& Avenir, vol. 2, 60, 176-194.

Ben Jelloun, Tahar. 1998. Le racisme expliqué à ma fille. Paris, Le Seuil.

BenAïssA, Aïcha. 2015. "Née en France. Histoire d'une jeune beur" in Luc Collès (éd.). L'immigration maghrébine dans la littérature française, anthologie France-Belgique (19532010). Bruxelles, EME éditions, 135-170.

BILICI, Faruk. 2003. “L'Islam en France sous l'Ancien Régime et la Révolution: attraction et répulsion" in Rives Méditerranéennes, 14, 17-37. <https://journals.openedition.org/ rives/406> [22/05/2021].

Boukhedenna, Sakinna. 2015. “Journal, Nationalité: immigré(e)” in Luc Collès (ed.). L'immigration maghrébine dans la littérature française, anthologie France-Belgique (19532010). Bruxelles, EME éditions, 117-170.

Bouvet Maisonneuve, Fatma. 2017. Une Arabe en France: Une vie au-delà des préjugés. Paris, Odile Jacob.

BURY, Laurent. 2010. L'Orientalisme victorien dans les arts visuels et la littérature. Grenoble, UGA Éditions (coll. Vers l'Orient).

Cheıкн, Yahya. 2010. "L'enseignement de l'arabe en France” in Langues et migrations | 1288.

Constantina, Badea. 2012. "Modèles d'intégration, identification nationale et attitudes envers les immigrés en France" in L'Année psychologique, 4, vol. 112, 575-592.

Costa Lascoux, Jacqueline.1991. "Le Droit à l'Ecole. L'expérience de l'éducation civique" in Droit et société: Le rapport des jeunes au Droit à l'Est et à l'Ouest, 19, 23-241.

FAsiki, Zainab. 2019. Hshouma. Corps et sexualité au Maroc. Paris, Massot.

FERAUD, Marie. 2015. “Anne ici, Sélima là-bas” in L'immigration maghrébine dans la littérature française, anthologie France- Belgique (1953-2010), (ed.) Luc Collès. EME éditions, 42-170. 
Anales de Filología Francesa, n. ${ }^{\circ}$ 29, 2021

LA FEMME MAGHRÉBINE ET FRANÇAISE D’ORIGINE MAGHRÉBINE CONFRONTÉE AUX PRÉJUGÉS DE LA...

Ferhati, Barkahom. 2007. "Les clôtures symboliques des Algériennes: la virginité ou l'honneur social en question" in Clio. Femmes, Genre, Histoire, 26, 169-180.

Gourdeau, Camille. 2015. "Une politique d'intégration au service des femmes étrangères" in Hommes \& migrations, 1311, 23-29.

Guerraoui, Zohra \& Sturm, Gesine. 2012. "Familles migrantes, familles en changement. Le paradigme de la complexité. L'exemple des familles d'origine maghrébine" in Devenir, vol. 24, 4, 289-299.

Hamel, Christelle. 2006. "La discrimination à l'encontre des femmes maghrébines en France" in Nouvelles questions féministes, vol. 25, 3, 124-134.

Hughes, Aaron W. 2013. Muslim Identities: An Introduction to Islam. New York, Columbia University Press.

JABBOUR, Rhéa E. 2013. Thèse de doctorat. La discrimination à raison de l'apparence physique (lookisme) en droit du travail français et américain: approche comparatiste. Paris, Université Panthéon-Sorbonne.

Jullien, François. 2021. Altérités: de l'altérité personnelle à l'altérité culturelle. Paris, Folio (coll. Essais).

KabBani, Rana. 1986. Europe's Myths of Orient. Indiana University Press.

Kulakowski, Christine. 2008. "Les voix des femmes" in Le livre Blanc de la femme migrante. Éditions Aouatif Zahim, 36-47.

Lacoste Dujardin, Camille. 2010. "Des femmes au Maghreb regards d'une ethnologue sur cinquante ans d'études et de recherches" in Hérodote. Revue de géographie et de géopolitique, $n^{\circ} 136,76-99$.

LAMchichi, Abderrahim. 2002. "l'islam de France à l'épreuve de la laïcité et du 'vivre ensemble" in Confluences méditerranée, $\mathrm{n}^{\circ} 41,141-160$.

Le Bras, Hervé. 2017, Malaise dans l'identité. Arles, Actes Sud.

Mahfoudh Draoui, Dorra. 2016. "Fatima Mernissi (1940-2015): la lutte pour un féminisme sans tutelle” in Nouvelles Questions Féministes, vol.35, n 2, 154-155.

Mernissi, Fatima. 1983. Sexe, idéologie, Islam. Paris, Tierce.

Mozzo Counil, Françoise. 1994. Femmes maghrébines en France: Mon pays, c'est ici, mon pays, c'est là-bas. Lyon, Chronique Sociale.

Manmane Guessous, Soumeya. 1991. Au-delà de toute pudeur: la sexualité féminine au Maroc. Casablanca, Eddif.

QRIBI, Abdelhak. 2017. “La femme d'origine maghrébine en immigration. Dynamiques identitaires, genre et personnalisation" in Éducation et socialisation, 44 | 2017: <http://journals. openedition.org/edso/2182> [23/05/2021]; DOI: 10.4000/edso.2182.

RAGI, Tariq. 2002. "Islam et laïcité" in Agora débats/jeunesses, Jeunes, engagement et démocratie, 30, 4-13. 
SiCARD, Frédéric. 2011. Enfants issus de l'immigration maghrébine: grandir en France. Paris, L'Harmattan.

Tersigni, Simona. 2001. “'Honneur maghrébin', différence culturelle et intégration. Variations sur quelques mots/maux des sciences sociales" in Confluences Méditerranée, vol. 39, $\mathrm{n}^{\circ} 4,55-65$.

Thomazo, Renaud. 2013. "La fleur de Lys" in Les grands symboles de l'Histoire de France, (ed.) Carine Girac-Marinier, Larousse, 14-15.

TouATI, Zeineb. 2012. “Travail des Maghrébines en France: spécificités et freins” in SociologieS: Dossiers, professions et métiers autour de la Méditerranée: <https://journals.openedition.org/sociologies/4028> [11/06/2021].

VASSBERG, Lilian M. 1997. “Immigration maghrébine en France: L'intégration des femmes" in The French Review. Vol.70, 5, 710-720.

WiJdAn, Ali. 2002. "Les femmes musulmanes: entre cliché et réalité" in Diogène, vol. 199, 3, 92-105.

YÁÑEz Solana, Manuel. 1995. El gran libro de los Nombres. Madrid, M. E. Editores. 
